ELECTRONIC RESEARCH ANNOUNCEMENTS

OF THE AMERICAN MATHEMATICAL SOCIETY

Volume 6, Pages 31-39 (April 24, 2000)

S $1079-6762(00) 00077-9$

\title{
GEOMETRY AND TOPOLOGY OF R-COVERED FOLIATIONS
}

\author{
DANNY CALEGARI \\ (Communicated by Walter Neumann)
}

\begin{abstract}
An $\mathbb{R}$-covered foliation is a special type of taut foliation on a 3manifold: one for which holonomy is defined for all transversals and all time. The universal cover of a manifold $M$ with such a foliation can be partially compactified by a cylinder at infinity, somewhat analogous to the sphere at infinity of a hyperbolic manifold. The action of $\pi_{1}(M)$ on this cylinder decomposes into a product by elements of $\operatorname{Homeo}\left(S^{1}\right) \times \operatorname{Homeo}(\mathbb{R})$. The action on the $S^{1}$ factor of this cylinder is rigid under deformations of the foliation through $\mathbb{R}$-covered foliations. Such a foliation admits a pair of transverse genuine laminations whose complementary regions are solid tori with finitely many boundary leaves, which can be blown down to give a transverse regulating pseudo-Anosov flow. These results all fit in an essential way into Thurston's program to geometrize manifolds admitting taut foliations.
\end{abstract}

\section{HoLONOMY}

1.1. Notation. Our notational convention in this paper will be that if $\Sigma$ is some structure or object on or in a manifold $M$, then $\widetilde{\Sigma}$ denotes the pullback of $\Sigma$ to the universal cover of $M$, which is consequently denoted by $\widetilde{M}$.

\subsection{Collating holonomy.}

Definition 1.1. A taut foliation $\mathcal{F}$ of a 3 -manifold $M$ is a codimension one foliation such that there is a circle in $M$ transverse to $\mathcal{F}$ which intersects every leaf.

For a taut foliation, every leaf is incompressible, and every transverse loop is homotopically essential by [9]. Furthermore, there is a metric on $M$ such that every leaf of $\mathcal{F}$ is a minimal surface by [10].

In this paper we will discuss a particular special class of taut foliations, known as $\mathbb{R}$-covered foliations.

Definition 1.2. An $\mathbb{R}$-covered foliation $\mathcal{F}$ is a taut, codimension one foliation of a 3-manifold $M$ such that the pulled-back foliation $\widetilde{\mathcal{F}}$ of $\widetilde{M}$ is the product foliation of $\mathbb{R}^{3}$ by horizontal $\mathbb{R}^{2}$ 's.

The definition of $\mathbb{R}$-covered given above is global in nature. It would be nice to characterize $\mathbb{R}$-covered foliations by some local property, but this seems to be very hard to do. It is easy to come up with examples of taut foliations which are not

Received by the editors May 7, 1999.

2000 Mathematics Subject Classification. Primary 57M50.

Key words and phrases. Foliations, laminations, 3-manifolds, geometrization, $\mathbb{R}$-covered, product-covered, group actions on $\mathbb{R}$ and $S^{1}$. 
$\mathbb{R}$-covered. For example, if $\mathcal{F}$ contains a compact leaf which is not the fiber of a fibration over $S^{1}, \mathcal{F}$ is not $\mathbb{R}$-covered.

$\mathbb{R}$-covered foliations have an intuitive description in terms of holonomy. Suppose we have a square $S$ transverse to $\mathcal{F}$ whose top and bottom sides are tangent to $\mathcal{F}$, and whose left and right sides are therefore transverse to $\mathcal{F}$. Then the intersection of $\mathcal{F}$ with the square $S$ induces a codimension 1 foliation of $S$, giving it the form of a product $I \times I$, where each vertical interval $I \times$ point is transverse to $\mathcal{F}$, and each horizontal interval point $\times I$ is tangent to a leaf of $\mathcal{F}$. This product structure allows one to define a homeomorphism from the left side of $S$ to the right side of $S$, by

$$
H_{[S]}:(t, 0) \rightarrow(t, 1)
$$

called the holonomy transport. One observes that this homeomorphism only depends on the homotopy class of $S$ through transverse squares with left and right sides fixed.

For a general pair of transversals $\tau, \tau^{\prime}$ and a homotopy class of path $\gamma$ from a point in $\tau$ to a point in $\tau^{\prime}$, one cannot always find a square $S$ as above containing $\tau$ and $\tau^{\prime}$ in its left and right sides, and $\gamma$ as a horizontal leaf, unless the foliation is $\mathbb{R}$-covered. This has as a consequence that there is a well-defined holonomy representation of $\pi_{1}(M)$ in $\operatorname{Homeo}(L)$, where $L$ denotes the leaf space of $\widetilde{\mathcal{F}}$. Of course, $L=\mathbb{R}$.

Theorem 1.1 (folklore). The holonomy groupoids over all leaves of an $\mathbb{R}$-covered foliation can be collated into a representation

$$
\rho_{H}: \pi_{1}(M) \rightarrow \operatorname{Homeo}(\mathbb{R})
$$

called the holonomy representation.

Philosophically, it might be reasonable to extend the definition to include foliations covered by the product foliation of $S^{2} \times \mathbb{R}$ by horizontal $S^{2}$ 's since these too have a holonomy representation, but conventionally one does not do so.

We make the simplifying assumption in what follows that our 3-manifold $M$ is orientable, and our foliation $\mathcal{F}$ is co-orientable. It follows that the holonomy representation acts by orientation-preserving homeomorphisms of the leaf space $\mathbb{R}$. This is not a serious restriction, since it can be achieved by passing to a finite cover.

\section{Confined LEAVES AND SLITHERINGS}

In [11, Thurston makes the following definition:

Definition 2.1. A manifold $M$ slithers over $S^{1}$ if there is a fibration $\phi: \widetilde{M} \rightarrow S^{1}$ such that $\pi_{1}(M)$ acts on this fibration by bundle maps. A slithering induces a foliation of $\widetilde{M}$ by the connected components of preimages of points in $S^{1}$ under the slithering map, and this descends to an $\mathbb{R}$-covered foliation of $M$.

By compactness of $M$ and $S^{1}$, it is clear that the leaves of $\widetilde{\mathcal{F}}$ stay within bounded neighborhoods of each other for a foliation $\mathcal{F}$ obtained from a slithering. That prompts us to define a confined leaf.

Definition 2.2. A leaf $\lambda$ of the pulled-back foliation $\widetilde{\mathcal{F}}$ of $\widetilde{M}$ is confined if there is a neighborhood $U$ of $\lambda$ in the leaf space $L$ such that for any $\mu$ in $U$ there is a $\delta$ 
with the property that

$$
\mu \subset N_{\delta}(\lambda)
$$

where $N_{\delta}(*)$ denotes the $\delta$-neighborhood of a set.

In [1] we show that this condition is symmetric. That is, we show

Theorem 2.1. For $\lambda, \mu$ leaves of $\widetilde{\mathcal{F}}$, where $\mathcal{F}$ is $\mathbb{R}$-covered, there exists $\delta$ such that $\mu \subset N_{\delta}(\lambda)$ iff there exists $\delta^{\prime}$ such that $\lambda \subset N_{\delta^{\prime}}(\mu)$.

It follows that the confined leaves of $\mathcal{F}$ (i.e. those whose covers in $\widetilde{\mathcal{F}}$ are confined) are an open subset of $M$, and the unconfined leaves are a minimal set. Having established this technical theorem, one can show that either every leaf is confined, or else the confined leaves of $\widetilde{\mathcal{F}}$ fall into a collection of pockets of uniformly bounded transverse width, and they can be "blown down" (i.e. collapsed) in a standard way. In fact, we have the dichotomy:

Corollary 2.2. Let $\mathcal{F}$ be an $\mathbb{R}$-covered foliation. Then after possibly blowing down some confined pockets of leaves, we may assume that every leaf is dense. If every leaf is dense, then either $\widetilde{\mathcal{F}}$ contains no confined leaves or $\mathcal{F}$ arises from a slithering of $M$ over $S^{1}$.

This dichotomy can be translated into information about the holonomy representation as follows:

Corollary 2.3. Let $\mathcal{F}$ be an $\mathbb{R}$-covered foliation. Then after possibly blowing down some confined pockets of leaves, the holonomy representation is one of the following two kinds:

1. $\rho_{H}\left(\pi_{1}(M)\right)$ is conjugate to a subgroup of $\left.\widetilde{\operatorname{Homeo}(} S^{1}\right)$, the universal central extension of $\operatorname{Homeo}\left(S^{1}\right)$.

2. For any two compact intervals $I, J \subset L$ there is an $\alpha \in \pi_{1}(M)$ such that

$$
\rho_{H}(\alpha)(I) \subset J .
$$

In [11] Thurston conjectured that for an atoroidal $M$, every $\mathbb{R}$-covered foliation should arise from a slithering. This would make our corollary quite uninteresting. However, this conjecture is false, and in [2] we construct many examples of $\mathbb{R}$ covered foliations of hyperbolic 3-manifolds which do not arise from slitherings.

\section{Topology at Infinity}

For $M$ atoroidal and not covered by $S^{2} \times S^{1}$, any taut foliation may be assumed to have leaves of exponential growth. That is, the leaves look coarsely like surfaces of negative curvature. The classical uniformization theorem has been generalized by Candel to this context, and in fact one knows from [4]:

Theorem 3.1 (Candel). Let $\mathcal{F}$ be a taut foliation of an atoroidal, irreducible 3manifold $M$. Then there is a metric on $M$ such that the induced metric on leaves has constant curvature -1 .

We say that such a foliation has hyperbolic leaves. In the sequel, we assume that our $\mathbb{R}$-covered foliation has hyperbolic leaves. In the universal cover, leaves of $\widetilde{\mathcal{F}}$ are isometric to copies of the hyperbolic plane $\mathbb{H}^{2}$, and therefore they each have a circle at infinity. For a leaf $\lambda$ of $\widetilde{\mathcal{F}}$, let $S_{\infty}^{1}(\lambda)$ denote its circle at infinity. 
In [7] it is shown that under very general circumstances, a foliation admits a harmonic measure. That is, a measure $m$ on the foliated manifold, such that for any measurable, leafwise $C^{\infty}$ function $f$, we have

$$
\int_{M} \Delta_{\mathcal{F}} f d m=0
$$

where $\Delta_{\mathcal{F}}$ denotes the leafwise Laplacian. The leafwise Laplacian is a generalization of the standard Laplacian for a Riemannian manifold. Informally, if we imagine that each leaf of our foliation is painted on either side with a perfect coat of insulating paint so that heat can only flow within a leaf and not transverse to it, then the leafwise Laplacian governs the constrained heat flow for this system, exactly as the usual Laplacian governs the heat flow for a Riemannian manifold. In a local coordinate patch, where $T \mathcal{F}$ is spanned by an orthonormal basis $\frac{\partial}{\partial x_{i}}$ for $1 \leq i \leq k$ and $\frac{\partial}{\partial x_{i}}$ for $k<i \leq n$ are transverse, the leafwise Laplacian looks like

$$
\Delta_{\mathcal{F}}=\sum_{i=1}^{k} \frac{\partial^{2}}{\partial x_{i}^{2}}+\text { higher order terms. }
$$

For foliations with every leaf dense, the measure $m$ disintegrates into the product of the leafwise Lebesgue measure with a measure on normal transversals which is infinitesimally harmonic, thought of as a function on the leaves. Using this structure, Thurston shows in [12] that exactly one of the following possibilities occurs:

- The transverse measure can be decomposed nontrivially as a sum of transverse measures $m_{h}+m_{i}$ where $m_{h}$ is harmonic and $m_{i}$ is invariant - that is, its value on a transversal is invariant under holonomy transport of that transversal.

- For each pair of leaves $\lambda, \mu$ which are distance $\epsilon$ apart at $p \in \lambda$, a random walk on $\lambda$ will shrink the distance between $\lambda$ and $\mu$ to 0 with probability $1-\delta$, where $\delta \rightarrow 0$ as $\epsilon \rightarrow 0$.

A random walk on $\mathbb{H}^{2}$ determines a unique point at infinity with probability 1 , so this shows that a subset of $S_{\infty}^{1}(\lambda)$ of visual measure close to $2 \pi$ (for small $\epsilon$ ) can be compared with a similar subset of $S_{\infty}^{1}(\mu)$.

These directions at infinity where leaves converge allow one to partially define a connection on the union of circles $\amalg S_{\infty}^{1}(\lambda)$. That is, one has a collection of transversals to the $S^{1}$ direction which allow us to identify subsets of $S_{\infty}^{1}(\mu)$ with $S_{\infty}^{1}(\lambda)$. These transversals are dense in any circle, and can be used to topologize the union of circles.

As a basis for this topology, take sets $U$ defined as follows: for a point $p \in S_{\infty}^{1}(\lambda)$, there are nearby transversals $\tau_{l}, \tau_{r}$ which run between $S_{\infty}^{1}\left(\lambda^{+}\right)$and $S_{\infty}^{1}\left(\lambda^{-}\right)$where $\lambda$ is between $\lambda^{+}$and $\lambda^{-}$in $L$. Then the points of $U$ are the points in the circles $S_{\infty}^{1}(\mu)$ for $\mu$ between $\lambda^{+}$and $\lambda^{-}$which are squeezed between the transverse points $\tau_{l} \cap \mu$ and $\tau_{r} \cap \mu$. These open sets generate a topology for which $C_{\infty}=\coprod S_{\infty}^{1}(\lambda)$ is homeomorphic to a cylinder. The transversals sit in this cylinder as a collection of vertical lines of various lengths, whose closure is the whole of $C_{\infty}$.

There is an alternate description of the topology on $C_{\infty}$. For each small transversal $\tau$ to $\widetilde{\mathcal{F}}$, the unit tangent bundle to $\widetilde{\mathcal{F}}$ restricts to a circle bundle over $\tau$, whose total space is a cylinder. Each point in this cylinder determines a unique point in $C_{\infty}$, by following the corresponding leafwise geodesic ray emanating from $\tau$ until it 
lands at a point in the circle at infinity of that leaf. This map is a homeomorphism for the topology on $C_{\infty}$ defined above.

As mentioned above, the collection of transversals sitting in this cylinder $C_{\infty}$ is like a partially defined connection, which one would like to extend to a complete connection and thereby $\left(\pi_{1}(M)\right.$-equivariantly) trivialize $C_{\infty}$ as a product. One cannot integrate along this partial connection for all time - one falls off the end of an integral curve. Nevertheless, one can define, canonically, a collection of trajectories which are integral curves of the partial connection where it is defined. These are simply the leftmost trajectories - those transversals to the circles in $C_{\infty}$ which rotate clockwise as much as possible without ever crossing any of the transversals defined by the harmonic measure. Starting from an initial point, the leftmost trajectory is defined for all time, but it is possible that two (initially distinct) leftmost trajectories may run into each other in finite time. However, using the property of $\rho_{H}\left(\pi_{1}(M)\right)$ that it can blow up any interval in $L$ as large as we like, one can find leftmost trajectories which are distinct for as long as required. Extracting a limit, one of the two possibilities must occur:

1. Leftmost trajectories never run into each other.

2. There is an invariant spine - a bi-infinite properly embedded $\operatorname{arc}$ in $C_{\infty}$ transverse to the $S^{1}$ 's which is invariant under the action of $\pi_{1}(M)$.

In [1] we show

Theorem 3.2. If $\mathcal{F}$ is an $\mathbb{R}$-covered foliation with hyperbolic leaves and $C_{\infty}$ contains an invariant spine, then (after blowing down) either $\mathcal{F}$ comes from a slithering, or $M$ is solv and $\mathcal{F}$ is the suspension of the stable or unstable foliation of an Anosov automorphism of a torus.

If $\mathcal{F}$ is the suspension of the stable or unstable foliation of an Anosov automorphism of a torus, then in fact $C_{\infty}$ is a product, and leftmost trajectories are distinct for all time.

Thurston shows in [11 that for foliations coming from a slithering, the universal cylinder is a product. In fact this is easy to see, since when every pair of leaves $\mu, \lambda$ in $\widetilde{\mathcal{F}}$ are a uniformly bounded distance apart, a geodesic on $\mu$ stays a bounded distance away from a unique geodesic on $\lambda$, allowing us to canonically identify all the circles at infinity. In short, we have

Theorem 3.3. Let $\mathcal{F}$ be an $\mathbb{R}$-covered foliation with hyperbolic leaves. Then the circles $S_{\infty}^{1}$ can be collated in a cylinder $C_{\infty}=S_{\text {univ }}^{1} \times \mathbb{R}$ such that $\pi_{1}(M)$ acts by elements of Homeo $\left(S^{1}\right) \times \operatorname{Homeo}(\mathbb{R})$.

If $M$ is not solv, and $\mathcal{F}$ with dense hyperbolic leaves does not come from a slithering, we say that $\mathcal{F}$ is ruffled. This name reflects the fact that for such a foliation, each pair of leaves in $\widetilde{\mathcal{F}}$ have a dense set of points at infinity where they are asymptotic to each other, and a dense set of points at infinity where they diverge from each other.

The proof that $C_{\infty}$ for a ruffled foliation is a product is very robust, and may be greatly generalized. For many invariant structures at infinity one can blow up the vertical direction by the action of $\pi_{1}(M)$ to show that the structure is either vertical, or else there is an invariant spine and $M$ is solv. An example shows how genuine laminations in $M$ interact with a ruffled foliation.

We begin with the definition of a genuine lamination (see [6]). 
Definition 3.1. A lamination in a 3-manifold is a foliation of a closed subset of $M$ by 2 dimensional leaves. The complement of this closed subset falls into connected components, called complementary regions. A lamination is essential if it contains no spherical leaf or torus leaf bounding a solid torus, and furthermore if $C$ is the closure (with respect to the path metric in $M$ ) of a complementary region, then $C$ is irreducible and $\partial C$ is both incompressible and end incompressible in $C$. Here an end compressing disk is a properly embedded $\left(D^{2}-\left(\right.\right.$ closed arc in $\left.\left.\partial D^{2}\right)\right)$ in $C$ which is not properly isotopic rel $\partial$ in $C$ to an embedding into a leaf. Finally, an essential lamination is genuine if it has some complementary region which is not an I-bundle.

Each complementary region falls into two pieces: the guts, which carry the essential topology of the complementary region, and the interstitial regions, which are just $I$ bundles over noncompact surfaces, which get thinner and thinner as they go away from the guts. The interstitial regions meet the guts along annuli. Ideal polygons can be properly embedded in complementary regions, where the cusp neighborhoods of the ideal points run up the interstitial regions as $I \times \mathbb{R}^{+}$. An end compressing disk is just a properly embedded ideal monogon which is not isotopic rel $\partial$ into a leaf.

In [6] many important properties of genuine laminations are established, including the fact that their leaves are incompressible.

Definition 3.2. A lamination of a circle $S^{1}$ is a closed subset of the space of unordered pairs of distinct points in $S^{1}$ such that no two pairs link each other.

Thinking of $S^{1}$ as the circle at infinity of $\mathbb{H}^{2}$, a lamination of $S^{1}$ gives rise to a lamination of $\mathbb{H}^{2}$, by joining each pair of points in $S^{1}$ by the unique geodesic in $\mathbb{H}^{2}$ connecting them. A lamination $\Lambda_{\text {univ }}$ of $S_{\text {univ }}^{1}$ invariant under the action of $\pi_{1}(M)$ determines a lamination in each leaf of $\widetilde{\mathcal{F}}$, and the union of these laminations sweep out a lamination $\widetilde{\Lambda}$ of $\widetilde{M}$ which, by equivariance of the construction, covers a lamination $\Lambda$ in $M$. By examining $\widetilde{\Lambda}$, one sees that $\Lambda$ is genuine.

Theorem 3.4. Let $\Lambda$ be a genuine lamination transverse to a ruffled foliation, so that the intersection of any leaf of $\widetilde{\Lambda}$ with any leaf of $\widetilde{\mathcal{F}}$ is a quasigeodesic in that leaf of $\widetilde{\mathcal{F}}$. Then $\Lambda$ arises from a $\pi_{1}(M)$-equivariant lamination $\Lambda_{\text {univ }}$ on $S_{\text {univ }}^{1}$.

If the intersection is not quasigeodesic in each leaf of $\widetilde{\mathcal{F}}$, we can nevertheless straighten out the intersections leafwise. If $\lambda$ is a leaf of $\widetilde{\Lambda}$ and $\mu$ is a leaf of $\widetilde{\mathcal{F}}$ which intersect in a properly embedded arc $\alpha \subset \mu$, then the two ends of $\alpha$ determine two subsets $\alpha^{ \pm}$of $S_{\infty}^{1}(\mu)$. We replace $\alpha$ by $\bar{\alpha}$ which runs between the most anticlockwise points in $\alpha^{ \pm}$. This straightens out $\Lambda$ to a new lamination $\bar{\Lambda}$, but in performing the straightening it is possible that we collapse some of $\Lambda$, and we do not necessarily know that our new lamination is isotopic to the old one.

\section{Regulating Vector fields}

Definition 4.1. A vector field $X$ transverse to an $\mathbb{R}$-covered foliation $\mathcal{F}$ is regulating if in the universal cover, every integral curve of $\widetilde{X}$ intersects every leaf of $\widetilde{\mathcal{F}}$ exactly once.

By analyzing the action of $\pi_{1}(M)$ on $S_{\text {univ }}^{1}$ defined in the previous section, following Thurston one can construct a pair of laminations $\Lambda_{\text {univ }}^{ \pm}$on $S_{\text {univ }}^{1}$ invariant under 
the action of $\pi_{1}(M)$. We show in [1] that any such invariant lamination has complementary regions which are finite-sided ideal polygons. As before, an invariant lamination on $S_{\text {univ }}^{1}$ determines a lamination in each leaf of $\widetilde{\mathcal{F}}$ by the construction outlined at the end of the previous section.

If $M$ is atoroidal, the pair of laminations bind each leaf of $\widetilde{\mathcal{F}}$; that is, they cut up the leaves into finite-sided geodesic polygons of bounded diameter. The combinatorics of this decomposition is constant as we vary from leaf to leaf, and it therefore allows us to equivariantly define identifications between leaves of $\widetilde{\mathcal{F}}$. This identification can be easily made on the intersections of $\Lambda^{+}$and $\Lambda^{-}$, and then inductively extended to the complementary intervals and polygons. This lets us canonically identify each leaf $\lambda$ of $\widetilde{\mathcal{F}}$ with a copy of $\mathbb{H}^{2}$ in a $\pi_{1}(M)$ equivariant way. The fibers of this identification give a 1-dimensional foliation transverse to $\widetilde{\mathcal{F}}$, and the tangent vectors to this foliation descend to a regulating vector field on $M$. One can arrange for this vector field to have some closed orbits, corresponding to the centers of the polygonal regions in each leaf. If $M$ is toroidal but $\mathcal{F}$ has hyperbolic leaves, the tori can be shown to be regulating, and therefore we can inductively decompose $M$ along regulating tori to show the following:

Theorem 4.1. An $\mathbb{R}$-covered foliation with hyperbolic leaves admits a regulating transverse vector field which can be chosen to have some closed orbits.

Corollary 4.2. If $M$ admits an $\mathbb{R}$-covered foliation with hyperbolic leaves, then $\widetilde{M}$ has the structure of a product $\mathbb{R}^{2} \times \mathbb{R}$ such that $\pi_{1}(M)$ acts by elements of $\operatorname{Homeo}\left(\mathbb{R}^{2}\right) \times \operatorname{Homeo}(\mathbb{R})$. Moreover, there are elements $\alpha \in \pi_{1}(M)$ such that $\rho_{H}(\alpha)$ has no fixed points, and is conjugate to a translation.

Following [13] and [8, one can collapse $\widetilde{M}$ to the intersection $\widetilde{\Lambda}^{+} \cap \widetilde{\Lambda}^{-}$to give a slightly different transverse regulating flow. The flow that one constructs is topologically pseudo-Anosov, as defined by Mosher in [8]. The issue of whether or not the flow is literally pseudo-Anosov comes down to a smoothability issue.

Combined with the result at the end of the previous section, we can see that the universal circle is stable under perturbations through $\mathbb{R}$-covered foliations:

Corollary 4.3. Let $\mathcal{F}_{t}$ for $-1<t<1$ be a 1-parameter family of $\mathbb{R}$-covered foliations. Then the actions of $\pi_{1}(M)$ on the universal circles of $\widetilde{\mathcal{F}}_{t}$ are all isomorphic.

Proof. If $\widetilde{\Lambda_{t}}$ is the lamination transverse to $\widetilde{\mathcal{F}_{t}}$ constructed from the action on $\left(S_{\text {univ }}^{1}\right)_{t}$, then for $s$ sufficiently close to $t, \widetilde{\Lambda_{t}}$ is transverse to $\widetilde{\mathcal{F}}_{s}$ and intersects it quasigeodesically. It follows that after straightening, $\widetilde{\Lambda_{t}}$ arises from an invariant lamination on $\left(S_{\text {univ }}^{1}\right)_{s}$. Since the endpoints of such laminations are dense, it follows that we can canonically and $\pi_{1}(M)$-equivariantly identify $\left(S_{\text {univ }}^{1}\right)_{s}$ with nearby $\left(S_{\text {univ }}^{1}\right)_{t}$, establishing the claim.

Finally, observe that the laminations constructed in $M$ have complementary regions which are finite-sided ideal polygon bundles over $S^{1}$. By a result of Gabai and Kazez in [5], this implies the following corollary:

Corollary 4.4. If $M$ admits an $\mathbb{R}$-covered foliation, then any homeomorphism $f$ : $M \rightarrow M$ homotopic to the identity is isotopic to the identity.

Remark 4.1. The methods of [1] have been used to produce analogous structures for taut foliations whose leaf spaces (in the universal cover) branch in at most one 
direction, and for certain taut foliations whose leaf spaces branch in either direction. These results are described in [3]. Finally we point out that some of the results in [1] have been obtained independently by S. Fenley.

We can summarize our results in the following flowchart:

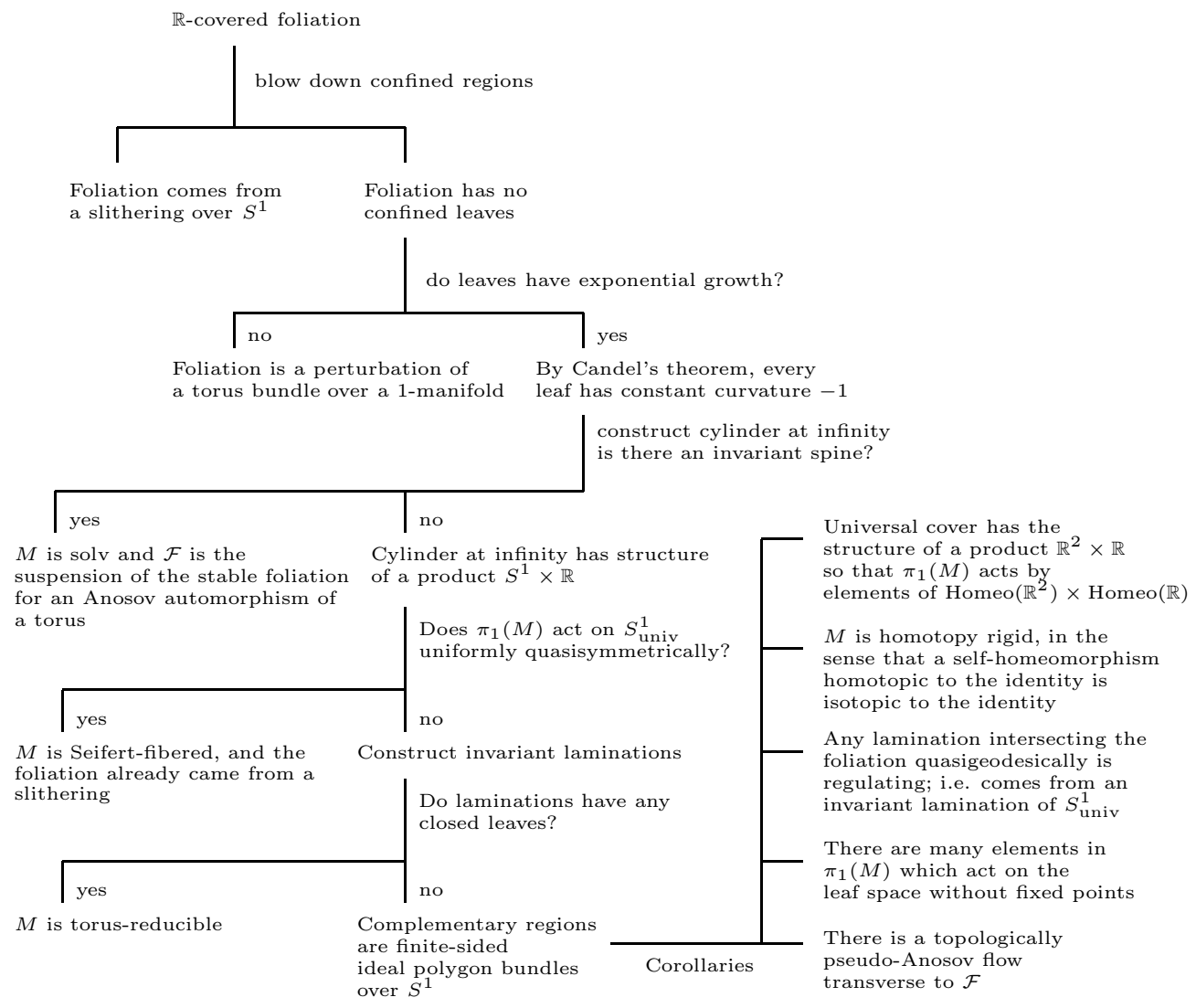

As remarked earlier, all the possibilities suggested by this flowchart actually occur, by the construction in 2 .

\section{REFERENCES}

[1] D. Calegari, The geometry of $\mathbb{R}$-covered foliations I, math.GT/9903173.

[2] D. Calegari, $\mathbb{R}$-covered foliations of hyperbolic 3-manifolds, Geometry and Topology 3 (1999), pp. 137-153. MR 2000c:57038

[3] D. Calegari, Foliations with one-sided branching, preprint.

[4] A. Candel, Uniformization of surface laminations, Ann. Sci. Ec. Norm. Sup. (4) 26 (1993), pp. 489-516. MR 94f:57025

[5] D. Gabai and W. Kazez, Homotopy, isotopy and genuine laminations of 3-manifolds, in Geometric Topology (ed. W. Kazez) proceedings of the 1993 Georgia International Topology Conference, Vol. 1, pp. 123-138. MR 98k:57026]

[6] D. Gabai and U. Oertel, Essential laminations in 3-manifolds, Ann. Math. (2) 130 (1989), pp. 41-73. MR 90h:57012

[7] L. Garnett, Foliations, the ergodic theorem and Brownian motion, J. Func. Anal. 51 (1983), pp. 285-311. MR 84j:58099

[8] L. Mosher, Laminations and flows transverse to finite depth foliations, Part I: Branched surfaces and dynamics, preprint. 
[9] S. Novikov, Topology of foliations, Trans. Mosc. Math. Soc. (1965), pp. 268-304. MR 34:824

[10] D. Sullivan, A homological characterization of foliations consisting of minimal surfaces, Comm. Math. Helv. 54 (1979), pp. 218-223. MR 80m:57022

[11] W. Thurston, 3-manifolds, foliations and circles I, math.GT/9712268.

[12] W. Thurston, 3-manifolds, foliations and circles II, preprint.

[13] W. Thurston, Hyperbolic structures on 3-manifolds II: Surface groups and 3-manifolds which fiber over the circle, math.GT/9801045.

Department of Mathematics, UC Berkeley, Berkeley, CA 94720

E-mail address: dannyc@math.berkeley.edu 